\title{
Dissecting the roles of Ephrin-A3 in malignant peripheral nerve sheath tumor by TALENs
}

\author{
ZHENGGUANG WANG, ZHENDONG LIU, BO LIU, GENGYAN LIU and SONG WU \\ Department of Orthopaedic Surgery, The Third Xiangya Hospital of Central South University, \\ Changsha, Hunan 410013, P.R. China
}

Received October 29, 2014; Accepted January 30, 2015

DOI: $10.3892 /$ or.2015.3966

\begin{abstract}
Malignant peripheral nerve sheath tumor (MPNST) is a rare and aggressive soft tissue sarcoma for which effective treatments have not yet been established due to poor understanding of its pathogenesis. Our previous study indicated that miR-210-mediated Ephrin-A3 (EFNA3) promotion of proliferation and invasion of MPNST cells plays an important role in MPNST tumorigenesis and progression. The purpose of the present study was to further investigate the roles of EFNA3 in MPNST. Constructed transcription activator-like effector nucleases (TALENs) and lentiviral vectors were transfected into MPNST ST88-14 (NF1 wild-type) and sNF96.2 (NF1 mutant type) cell lines to obtain gain- and loss-of-function cell lines for the EFNA3 function study. The results showed that the knockout of ENFA3 increased cellular viability and invasiveness of the MPNST cells. However, the adhesion ability of MPNST cells was enhanced or inhibited when EFNA3 was overexpressed or knocked out, respectively. It was also observed that knockout of EFNA3 significantly decreased the expression of phosphorylated FAK (p-FAK) and the tumor necrosis factor $\alpha$ (TNF- $\alpha$ ) compared to that in the control cells, yet the expression of phosphatidylinositol 3-kinase (PI3K), GTPase, integrins, vascular endothelial growth factor (VEGF) and hypoxia-inducible factor $1 \alpha$ (HIF- $\alpha$ ) increased significantly. Inversely, overexpression of EFNA3 significantly increased the expression of p-FAK and TNF- $\alpha$ compared to that in the control cells, yet the expression of PI3K, GTPase, integrins, VEGF and HIF- $\alpha$ decreased significantly. The results indicated that EFNA3 serves as a tumor suppressor in MPNST cells and it may play a critical role in the focal adhesion kinase (FAK) signaling and VEGF-associated tumor angiogenesis pathway. These findings may not only facilitate
\end{abstract}

Correspondence to: Professor Song Wu, Department of Orthopaedic Surgery, The Third Xiangya Hospital of Central South University, 138 Tongzipo Road, Changsha, Hunan 410013, P.R. China E-mail: xiangyawusong@163.com

Key words: malignant peripheral nerve sheath tumor, Ephrin-A3, microRNA-210, pathogenesis the better understanding of MPNST pathogenesis, but also suggest EFNA3 as a promising target for MPNST treatment.

\section{Introduction}

Malignant peripheral nerve sheath tumor (MPNST), typically arising from Schwann cells of peripheral nerve sheaths (1-3), is known as the most aggressive peripheral nerve malignant tumors. MPNST is the main soft tissue malignancy associated with neurofibromatosis type 1 (NF1) (4). It has been reported that $\sim 80 \%$ of MPNSTs are pathologically indicated as high-grade tumors (2). Furthermore, studies have shown that $20-50 \%$ of patients with MPNST also have NF1 and $10 \%$ of patients with NF1 will finally suffer from MPNST $(1,4,5)$. Traditional treatment methods including surgical resection, chemotherapy and radiotherapy can not obtain an ideal curative effect mainly due to the aggressive growth and metastasis of MPNST. Therefore, it is urgent to clarify the underlying molecular mechanism of MPNST for developing new molecular therapeutic tools.

Eph receptors represent the largest family of receptor tyrosine kinases (RTKs), which are capable of recognizing signals from the cell environment and influencing cell-cell interaction and cell migration (6-8). Ephrins are the ligands to Eph receptors and they stimulate bi-directional signaling of the Eph-ephrin axis. Ephrin-A3 (EFNA3) is one of the ephrin ligands which could bind to EphA2, EphA3, EphA5, EphA7, EphA8 and more poorly to EphA4. It is not only expressed in skeletal muscle, spleen, thymus, prostate, testis, ovary, small intestine and peripheral blood leukocytes, but is also present in neuroblastomas, neural cancers and leukemias. The dysregulated expression of EFNA3 has been observed in many types of human cancer. The expression level of EFNA3 was found to be upregulated 26-fold in squamous cell lung carcinoma, 3.8-fold in liver cancer, 1.6-fold in colon cancer and downregulated 2.6-fold in kidney carcinoma (9-12). Our previous study also showed that mRNA expression levels of EFNA3 were significantly decreased in MPNST cell lines (1). However, the molecular mechanism by which EFNA3 mediates MPNST cells is still unknown.

MicroRNAs (miRs), a kind of endogenous non-coding RNAs, can serve as endogenous agents for RNA interference. Growing evidence indicates that miRs deregulation is closely related to certain pathological processes including 
tumorigenesis. miRs can act as tumor suppressors or oncogenes depending on their targets (13). Among these functional miRs, miR-210 is frequently upregulated in various types of cancer, such as glioblastoma (14), clear cell renal cell carcinoma (15), lung (16) and breast cancer $(17,18)$. miR-210 may play an oncogenic role in cancer initiation and progression via regulating cellular growth, apoptosis, migration and invasion (19,20). In MPNST, Presneau et al identified 16 significantly differentially expressed miRs in MPNST relatively to neurofibromas. Of these, miR-210 was identified with increased expression (21). In addition, our previous study (1) indicated that miR-210 reduced the expression of its target gene EFNA3 and stimulated growth and invasion of MPNST. Accordingly, EFNA3 plays a role in MPNST progression and miR-210 acts as an oncogene. However, the relationship between miR-210 and EFNA3 in MPNST cells requires further investigation.

Overexpression and knockout of a specific gene are the crucial strategies for gene function study. Transfection strategies, which along with plasmid or lentiviral vectors, are known as the powerful methods for overexpression of a specific gene. Various technical tools have been developed to probe the functions of genes, yet their application has been limited by low efficacy and specificity (22). Recently, transcription activator-like effector nucleases (TALENs) emerged as a novel promising tool for gene function analysis. TALENs are artificial restriction enzymes generated by fusing a TAL effector DNA binding domain to a DNA cleavage domain. Transcription activator-like effectors (TALEs) can be quickly engineered to bind practically any desired DNA sequence (23). When these restriction enzymes are introduced into cells, they can be used for gene knockout. In the present study, TALENs and lentiviral transfection strategies were applied to dissect the roles of EFNA3 in MPNST cells and to reveal correlation between EFNA3 and miR-210. The present study may facilitate better understanding of MPNST pathogenesis and the development of potential therapeutic targets for MPNST.

\section{Materials and methods}

Cell culture. MPNST ST88-14 (NF1 wild-type) and sNF96.2 (NF1 mutant type) cell lines were purchased from the China Center for Type Culture Collection (CCTCC; Wuhan, China). All the cells were cultured in Dulbecco's modified Eagle's medium (DMEM) supplemented with $10 \%$ fetal bovine serum (FBS; Life Technologies, Carlsbad, CA, USA), $100 \mathrm{IU} / \mathrm{ml}$ penicillin and $100 \mu \mathrm{g} / \mathrm{ml}$ streptomycin sulfate at $37^{\circ} \mathrm{C}$ in a humidified incubator containing $5 \% \mathrm{CO}_{2}$.

Antibodies. Antibodies of EFNA3 and $\beta$-actin were obtained from Santa Cruz Biotechnology (Dallas, TX, USA). Antibodies of focal adhesion kinase (FAK), phosphorylation FAK (p-FAK), PI3K, integrins, TNF- $\alpha$ and HIF- $1 \alpha$ were purchased from ImmunoWay Biotechnology (Newark, DE, USA). Antibodies of GTPase and VEGF were purchased from Abzoom (Dallas, TX, USA).

Real-time RT-PCR. Total RNA was extracted from cells (MPNST cell line ST88-14, T265p21, sNF96.2, YST-1 and MPNST-14 cells were purchased from CCTCC or Schwann cells separated from human NF1 neurofibroma tissues) with TRIzol reagent (Life Technologies) following the manufacturer's instructions. The expression of EFNA3 mRNA was detected by real-time RT-PCR using the standard SYBR-Green RT-PCR kit (Takara, Otsu, Japan) following the manufacturer's instructions. The specific primer pairs are as follows: EFNA3 sense, 5'-CTTGTGGCTCTGGTAATGTTTGG-3' and antisense, 5'-GAGGAGGACGTGCTTATTGCTGT-3'; $\beta$-actin as an internal sense, 5'-AGGGGCCGGACTCGTCATACT-3' and antisense, 5'-GGCGGCACCACCATGTACCCT-3'. The relative expression of the gene mRNA was quantified using the GraphPad Prism 4.0 software (GraphPad Software, San Diego, CA, USA) and the $2^{-\Delta \Delta \mathrm{Ct}}$ method (24).

Design and construction of TALENs. TAL effector DNA binding domains were designed and constructed based on TAL Effector Nucleotide Targeter 2.0 (25). The sites: gggaaaccggcatgcggt (left) and ccccgactcactgctggt (right) were chosen. According to Sanjana et al (26), the binding pairs EFNA3-L and EFNA3-R were, respectively, assembled into the pTALEN-v2-L and pTALEN-v2-R backbones, yielding pTALEN-EFNA3-L and pTALEN-EFNA3-R.ST88-14 and sNF96.2 cells were transfected with a mixture of pTALEN-EFNA3-L, pTALEN-EFNA3-R (untreated cells were used as a control). Cells were trypsinized and resuspended after transfection for 4 days. The transfected cells were expanded. To confirm the disruption of EFNA3, western blotting and PCR were performed. Whole cell extracts were analyzed by western blotting, and the targeted exon was PCR-amplified from genomic DNA isolated from individual clones. The following day, the medium was refreshed and grown for an additional $24 \mathrm{~h}$ prior to harvesting for further analysis.

Lentiviral transfection. The Lv-EFNA3 and Lv-NC lentiviral suspension was purchased from GeneChem (Shanghai, China). The titer of the lentiviral vectors was $2 \times 10^{10}$ titer units (TU)/ml. The ST88-14 and sNF96.2 cells were plated and cultured in 6 -well plates until cell fusion reached $60-70 \%$. Then, $2.5 \times 10^{4}$ TU/well Lv-EFNA3 or Lv-NC lentivirus was added to the cells under MOI values of 50. To confirm the effect of the lentivirus on the expression of EFNA3 gene, PCR and western blotting was performed to determinate the $\mathrm{mRNA}$ and protein levels of EFNA3 in the ST88-14 and sNF96.2 cells after infection with lentivirus for 6 days. The transfected cells were expanded and harvested for further analysis.

Cell viability assay. MPNST ST88-14 and sNF96.2 cells transfected with pTALEN-EFNA3-L, pTALEN-EFNA3-R and pre-miR-210 or pre-miR-210 in exponential growth were plated at a final concentration of $2 \times 10^{3}$ cells/well in 96-well plates. The viability of the cells was evaluated by an MTT assay after 24, 48, 72 and $96 \mathrm{~h}$ of seeding. The optical density at $570 \mathrm{~nm}$ (OD570) of each well was measured with an ELISA reader (ELX-800 type; BioTek, Winooski, VT, USA).

Cell invasion assay. The cell invasion assay was performed using a Cell Invasion Assay kit (Chemicon International, Temecula, CA, USA) according to the manufacturer's guidelines. Briefly, ST88-14 and sNF96.2 cells transfected with pTALEN-EFNA3-L and pTALEN-EFNA3-R or pre-miR-210 
A

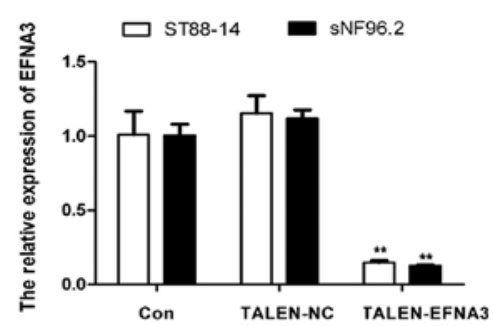

B
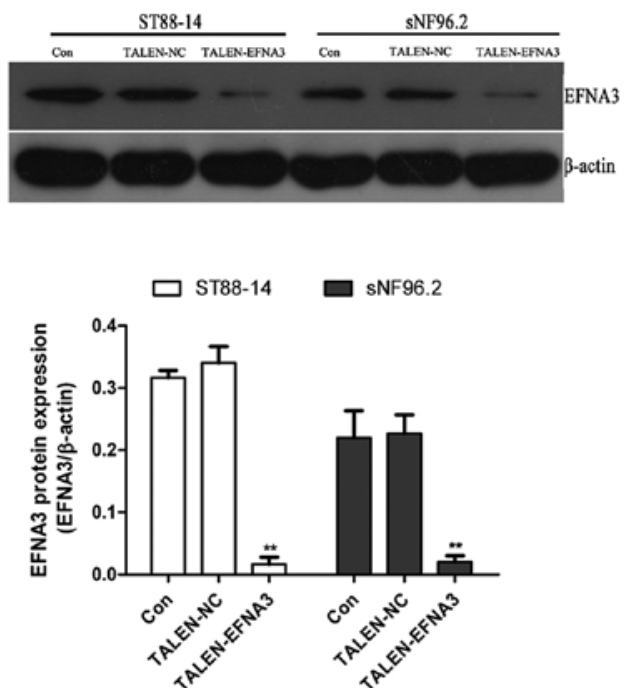

C

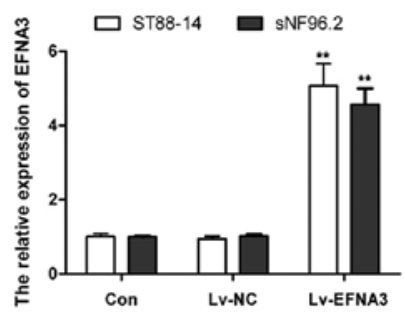

D
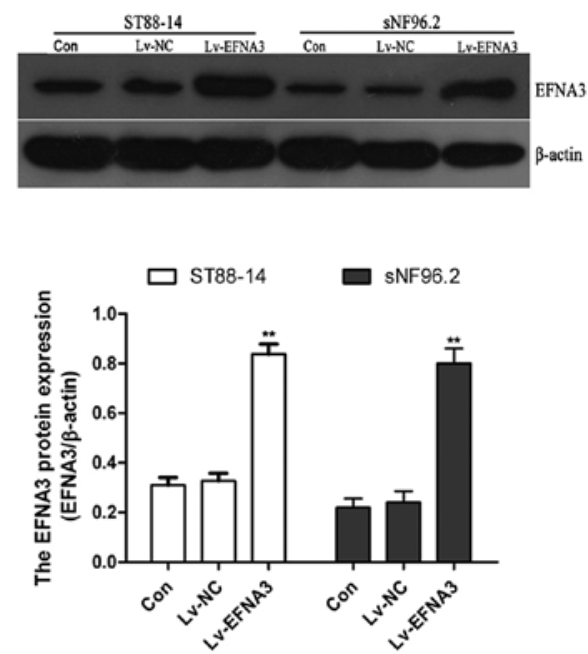

Figure 1. (A) RT-PCR detection of EFNA3 mRNA expression after transfection with EFNA3-TALENs; (B) western blotting detection of EFNA3 protein expression after transfection with EFNA3-TALENs; (C) RT-PCR detection of EFNA3 mRNA expression after transfection with Lv-EFNA3 or Lv-NC; (D) western blotting detection of EFNA3 protein expression after transfection with Lv-EFNA3 and Lv-NC. ** P<0.01 vs. con. EFNA3, Ephrin-A3; TALENs, transcription activator-like effector nucleases.

or pTALEN-EFNA3-L, pTALEN-EFNA3-R and pre-miR-210 and their corresponding negative control was placed in the upper compartment of the chambers, and DMEM containing $10 \%$ FBS was added in the lower chambers. After $24 \mathrm{~h}$ of incubation at $37^{\circ} \mathrm{C}$, cells on the upper face of the membrane were scraped using a cotton swab and cells on the lower face were fixed, stained and observed under a microscope. Then the dye on the membrane was dissolved with $10 \%$ acetic acid, dispensed into 96 -well plates $(150 \mu \mathrm{l} /$ well $)$, and the optical density at $570 \mathrm{~nm}$ (OD570) of each well was measured with an ELISA reader (ELX-800 type).

Cell adhesion assay. Adhesion was assayed by plating cells in DMEM on 96-well plates pre-coated with bovine serum albumin (BSA) as a control or $20 \mu \mathrm{g} / \mathrm{ml}$ fibronectin (FN) (both from Life Technologies), respectively. Cells were pre-treated overnight with MPNST cells and were allowed to adhere for $2 \mathrm{~h}$. Wells were washed, fixed with $4 \%$ paraformaldehyde and stained with crystal violet (Life Technologies). Adhered cells were counted under a microscope (AE31 type; Motic, HK, China) in five fields.

Western blotting. Cells were lysed in cell lysate, and then centrifuged at $12,000 \mathrm{x} \mathrm{g}$ for $20 \mathrm{~min}$ at $4^{\circ} \mathrm{C}$. The supernatant was collected and denatured. Proteins were separated in $10 \%$ SDS-PAGE and blotted onto polyvinylidene difluoride membrane (PVDF). The PVDF membrane was treated with
TBST containing $50 \mathrm{~g} / \mathrm{l}$ skimmed milk at room temperature for $4 \mathrm{~h}$, followed by incubation with the primary antibodies of EFNA3, FAK, p-FAK, PI3K, GTPase, integrins, VEGF, TNF- $\alpha, \mathrm{HIF}-1 \alpha$ and $\beta$-actin, respectively, at $37^{\circ} \mathrm{C}$ for $1 \mathrm{~h}$. Membranes were rinsed and incubated for $1 \mathrm{~h}$ with the correspondent peroxidase-conjugated secondary antibodies. Chemiluminent detection was performed with the ECL kit (Pierce Chemical Co., Rockford, IL, USA).

Statistical analysis. Data are expressed as mean \pm SD from at least three separate experiments. Statistical analysis was carried out using SPSS 15.0 software. The difference between the two groups was analyzed by the Student's t-test. A value of $\mathrm{P}<0.05$ was considered to indicate a statistically significant result.

\section{Results}

Knocked out or overexpressed EFNA3 in ST88-14 and sNF96.2 cells. In the present study, ST88-14 and SNF96.2 cells were selected to investigate the functions of EFNA3 in MPNST. TALENs are emerging as a new powerful technique in the field of targeted genome engineering. TALENs were applied for knockout of EFNA3 gene in MPNST cells. After transfection with pTALEN-EFNA3-L and pTALEN-EFNA3-R, the expression levels of EFNA3 mRNA and protein in ST88-14 and SNF96.2 cells were analyzed by RT-PCR and western 

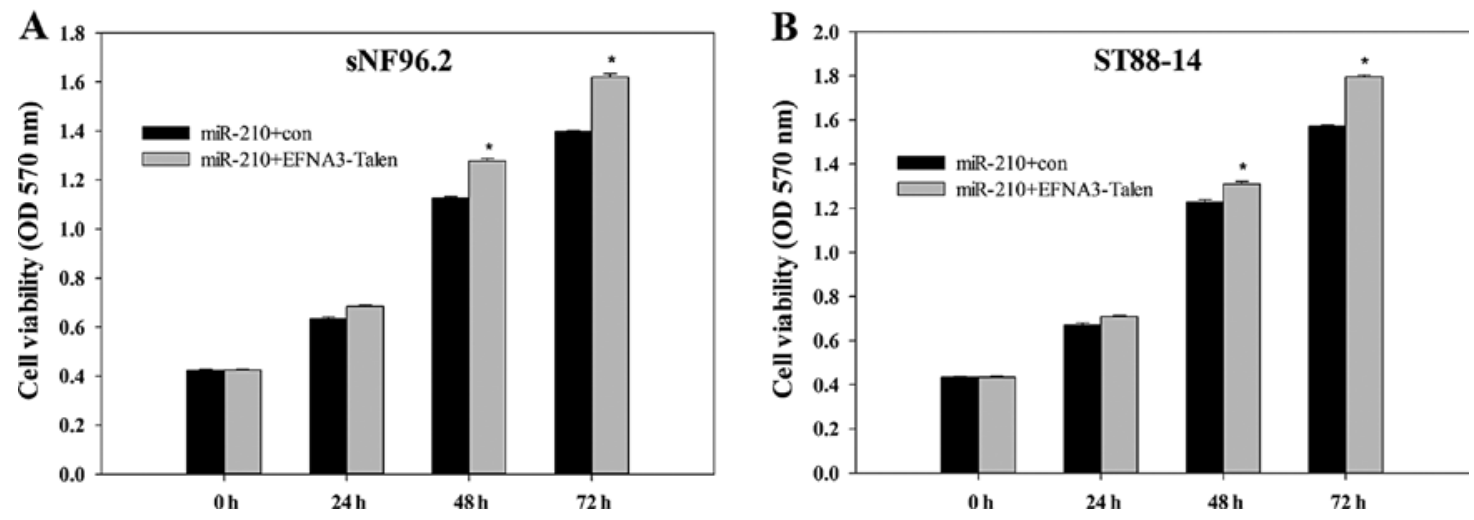

Figure 2. MTT assay was performed to determine the viability of (A) MPNST sNF96.2 and (B) ST88-14 cells in which miR-210 were firstly overexpressed and EFNA3 was further knocked out. ${ }^{*} \mathrm{P}<0.05$ vs. mir-210+con. MPNST, malignant peripheral nerve sheath tumor.
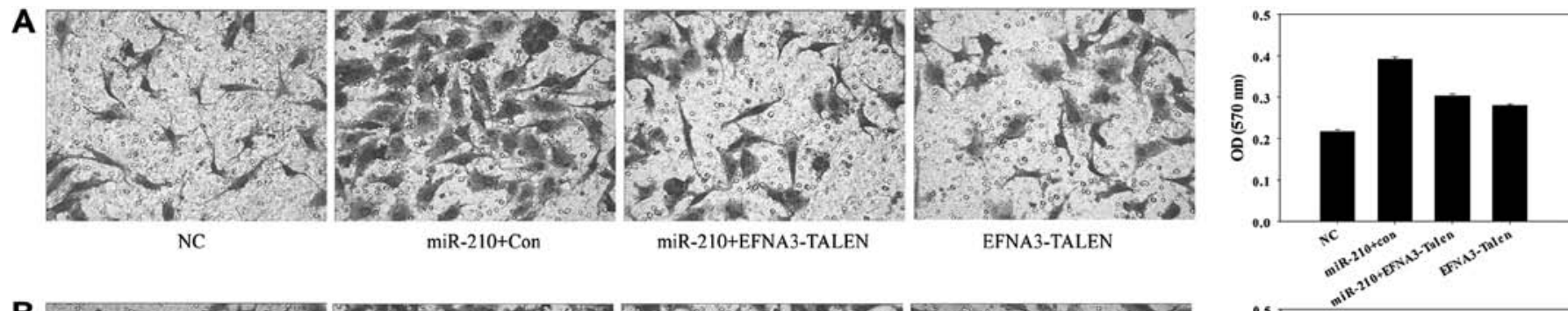

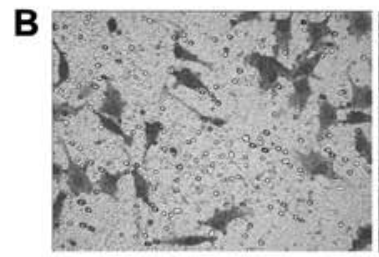

$\mathrm{NC}$

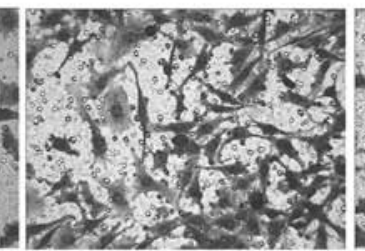

miR-210+Con

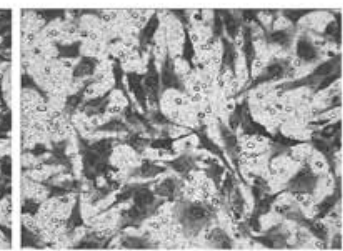

miR-210+EFNA3-TALEN

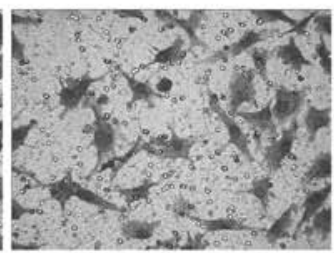

EFNA3-TALEN

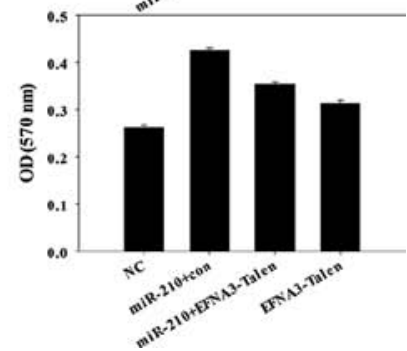

Figure 3. Transwell assay showed that knockout of EFNA3 or overexpression of miR-210 or both knockout of EFNA3 and overexpression of miR-210 all strongly increased the invasiveness of (A) sNF96.2 and (B) ST88-14 cells. Each group is significantly different from each other (P<0.05). EFNA3, Ephrin-A3.

blotting. The expression of EFNA3 mRNA and protein were detected (Fig. 1A and B). These results suggested that EFNA3 gene was effectively knocked out by EFNA3-TALENs in ST88-14 and sNF96.2 cells.

EFNA3 was also overexpressed by lentiviral transfection. After transfection with lentiviral recombinant vectors, RT-PCR and western blotting were performed to analyze the expression of EFNA3 mRNA and protein. As shown in Fig. 1C and D, the expression of EFNA3 mRNA and protein increased significantly in transfected LV-EFNA3 cells compared with transfected LV-NC vector and control cells. The above indicated that EFNA3 gene was overexpressed effectively by the LV-EFNA3 transfected in ST88-14 and sNF96. 2 cells.

Effect of EFNA3 on the viability of MPNST cells. MTT assay, generally applied to detect the viability of the cellse, was employed to investigate the effect of EFNA3 on the viability of ST88-14 and sNF96.2 cells. MPNST cells, with the EFNA3 gene present or knocked out, was transfected with pre-miR-210. Subsequently, the cell viabilities were evaluated every $24 \mathrm{~h}$ for 3 days. As shown in Fig. 2A and B, although the MPNST cells were all transfected with pre-miR-210, the viabilities still increased in ST88-14 and sNF96.2 cells when EFNA3 was knocked out. This suggested that the knockout of EFNA3 promoted the viability of the MPNST cells.

Effect of EFNA3 on invasiveness of MPNST cells. The rising invasiveness is an important feature of malignant tumors. The effects of EFNA3 on invasiveness of MPNST ST88-14 and sNF96.2 cells were examined by a Transwell assay. The results showed that knockout of EFNA3 by introduction of pTALEN-EFNA3-L and pTALEN-EFNA3-R, or overexpression of miR-210 by introduction of pre-miR-210, both strongly increased the invasiveness of ST88-14 and sNF96.2 cells (Fig. 3A and B). It was also noted that the overexpression of miR-210 had more power in increasing the invasiveness of the MPNST cells than that of the knockout of EFNA3. In addition, the MPNST cells with both knockout EFNA3 and overexpression of miR-210 increased the invasiveness of the cells as well. As presented in Fig. 3, the enhancement degree of invasiveness in MPNST cells with both the knocked out EFNA3 and overexpressed miR-210 were greater than that of EFNA3 knockout MPNST cells, but weaker than that of 

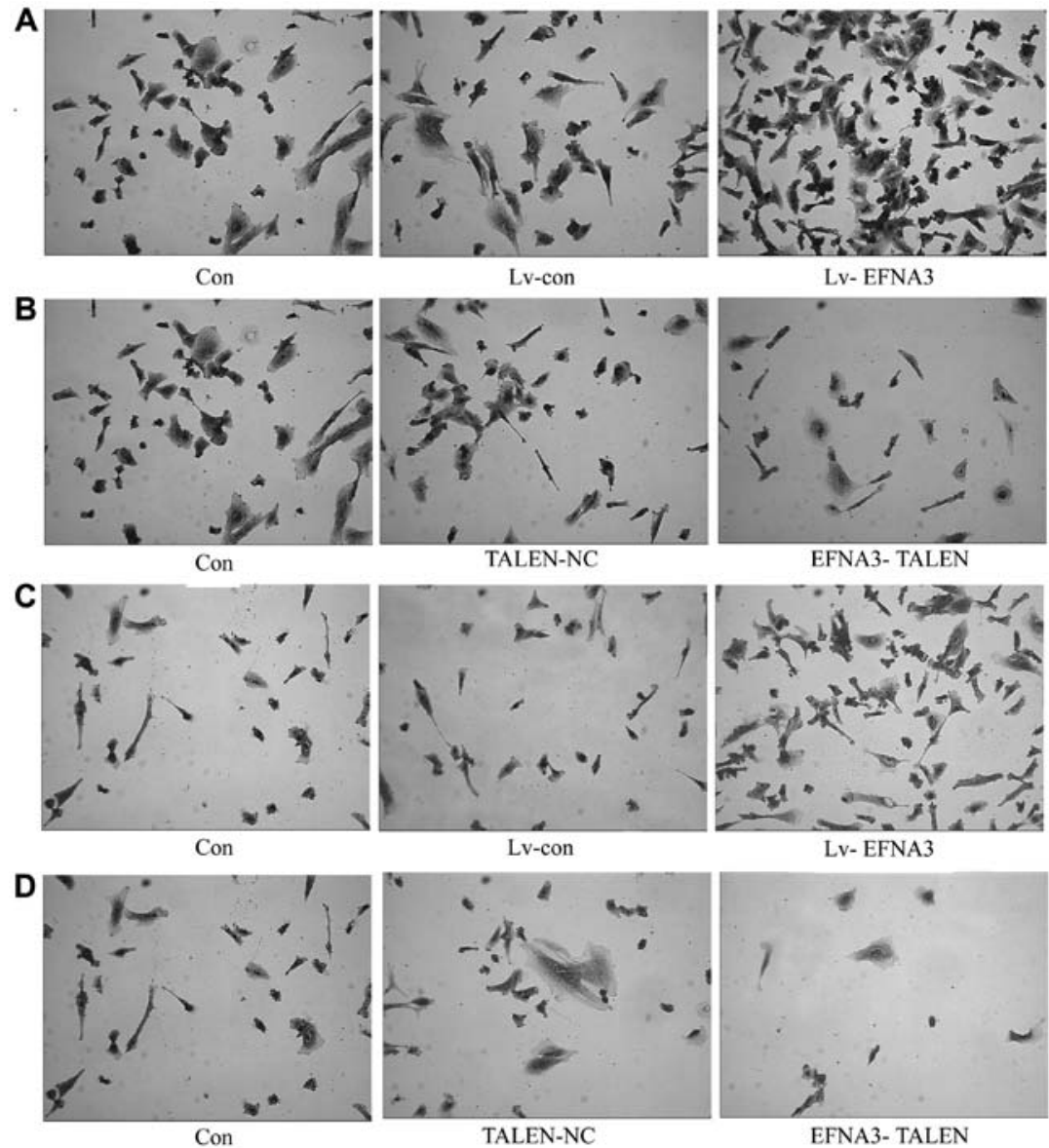

Figure 4. Cell adhesion assay showed that overexpression of EFNA3 effectively increased the adhesion capacity of (A) ST88-14 and (C) sNF96.2 cells, while the knockout of EFNA3 by TALENs significantly decreased the adhesion capacity of (B) ST88-14 and (D) sNF96.2 cells. EFNA3, Ephrin-A3; TALENs, transcription activator-like effector nucleases.

A
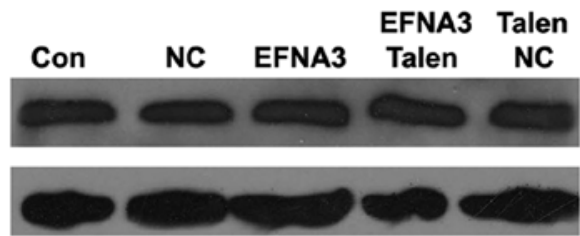

FAK

p-FAK

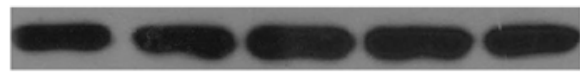

$\beta$-actin

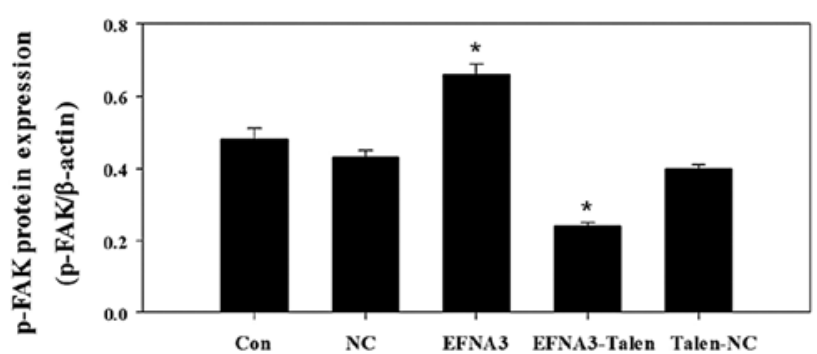

B
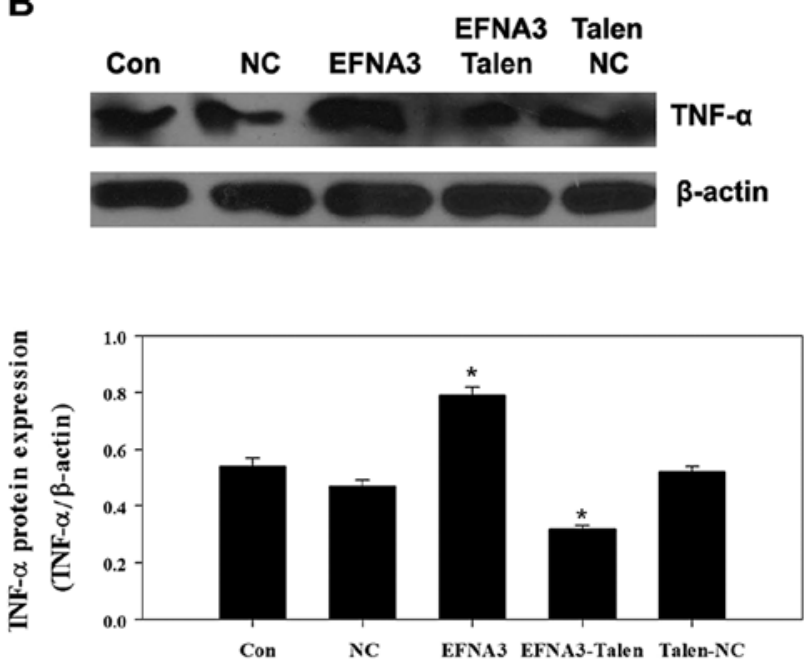

Figure 5. (A and B) Expression levels of FAK, p-FAK and TNF- $\alpha$ in ST88-14 cells infected with Lv-EFNA3, EFNA3-TALENs or TALENs-NC were detected by western blot analysis. "P<0.05 vs. con. FAK, focal adhesion kinase; p-FAK, phosphorylation FAK; TNF- $\alpha$, tumor necrosis factor $\alpha$; TALENs, transcription activator-like effector nucleases.

miR-210 overexpressed MPNST cells, suggesting that EFNA3 had a negative effect on the invasiveness of the MPNST cells. Furthermore, EFNA3 may not be the only target gene of miR-210.
Effect of EFNA3 on adhesion of MPNST. The adhesion capability of cancer cells is closely related with metastasis. To investigate the effect of EFNA3 on adhesion of MPNST cells, EFNA3 in ST88-14 and sNF96.2 cells was knocked out by 

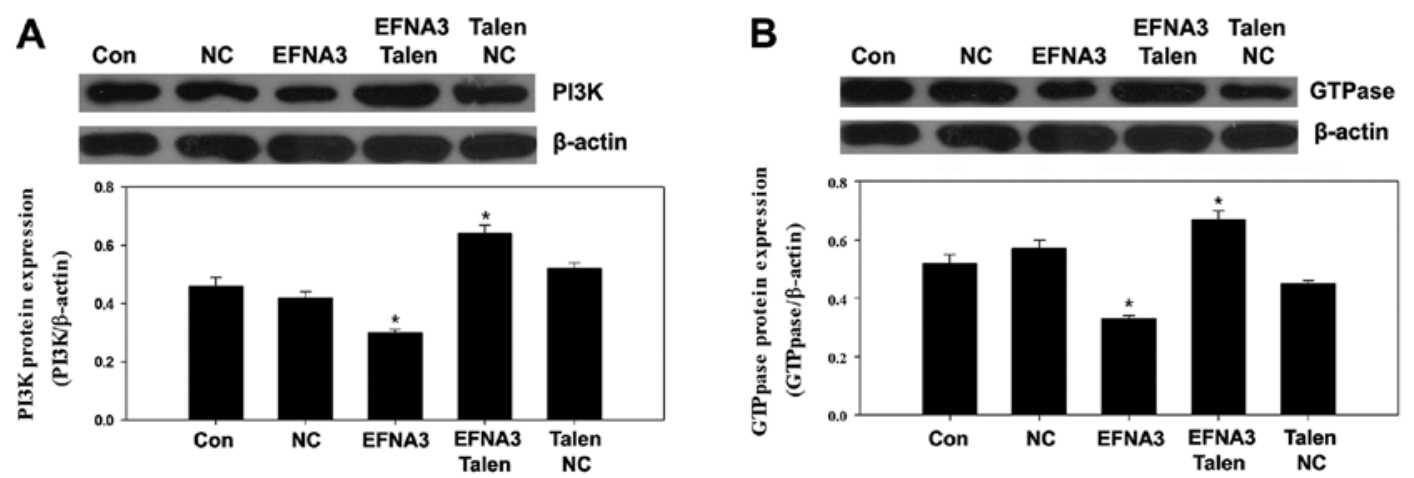

Figure 6. (A) Protein expression levels of PI3K in ST88-14 cells infected with Lv-EFNA3, EFNA3-TALENs or TALENs-NC were detected by western blot analysis. "P<0.05 vs. con. (B) Protein expression levels of GTPase in ST88-14 cells infected with Lv-EFNA3, EFNA3-TALENs or TALENs-NC were detected by western blot analysis. ${ }^{*} \mathrm{P}<0.05$ vs. con.

A
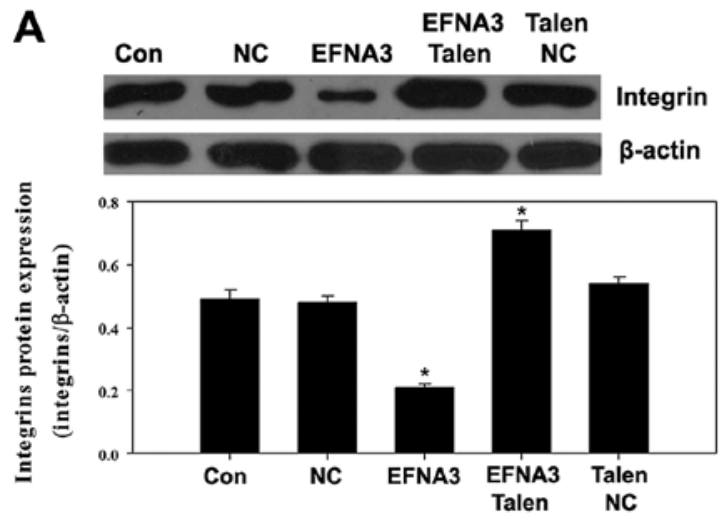

B
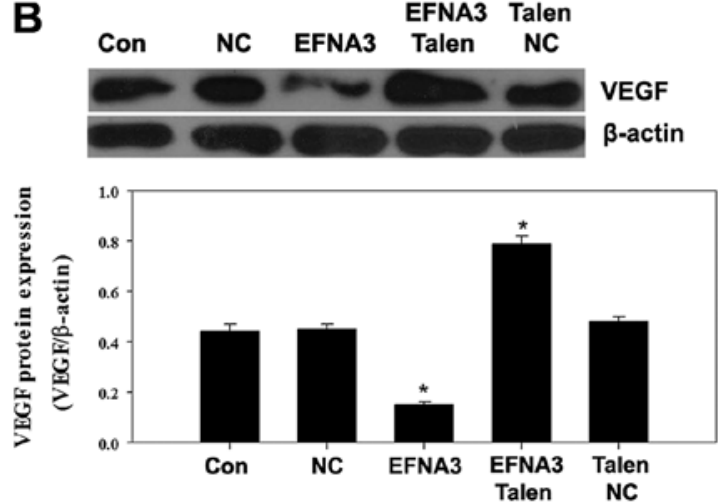

Figure 7. (A) Protein expression levels of integrins in ST88-14 cells infected with Lv-EFNA3, EFNA3-TALENs or TALENs-NC were detected by western blot analysis. "P<0.05 vs. con. (B) Protein expression levels of VEGF in ST88-14 cells infected with Lv-EFNA3, EFNA3-TALENs or TALENs-NC were detected by western blot analysis. "P<0.05 vs. con. TALENs, transcription activator-like effector nucleases; VEGF, vascular endothelial growth factor.

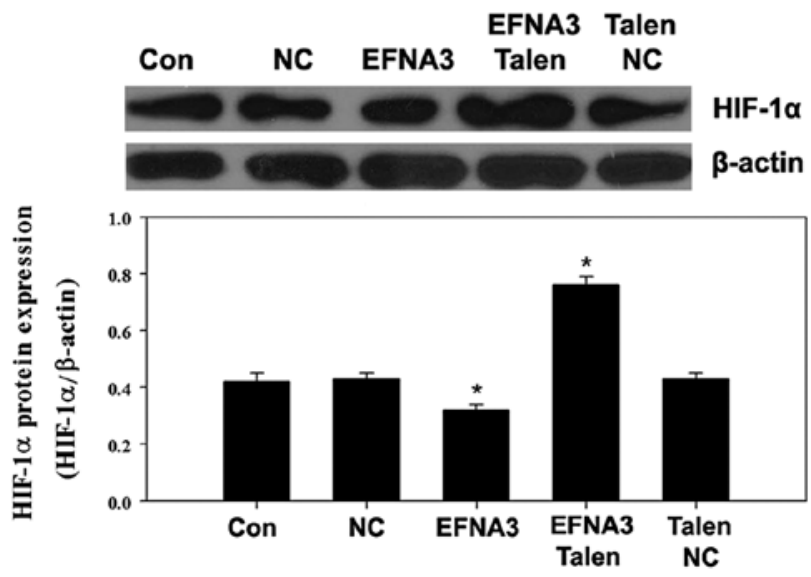

Figure 8. Protein expression levels of HIF-1 $\alpha$ in ST88-14 cells infected with Lv-EFNA3, EFNA3-TALENs or TALENs-NC were detected by western blot analysis. ${ }^{*} \mathrm{P}<0.05$ vs. con. HIF- $1 \alpha$, hypoxia-inducible factor $1 \alpha$; TALENs, transcription activator-like effector nucleases.

TALENs that also overexpressed EFNA3 through Lv-EFNA3 transfection. Our data showed that the adhesion capability of MPNST cells was inhibited after knocking out EFNA3, while overexpression of EFNA3 enhanced the adhesion capability of both ST88-14 and sNF96.2 cells (Fig. 4A-D). These findings suggest that EFNA3 inhibits MPNST metastasis by promoting the adhesion of MPNST cells.

Molecular mechanism underlying the role of EFNA3 in tumor angiogenesis. To explore the potential molecular mechanisms underlying EFNA3-induced tumor angiogenesis, the expression of some protein members of FAK signaling pathway including FAK, p-FAK, phosphatidylinositol 3-kinase (PI3K), GTPase as well as integrins and angiogenic factors including vascular endothelial growth factor (VEGF), tumor necrosis factor $\alpha(\mathrm{TNF}-\alpha)$ and hypoxia-inducible factor $1 \alpha(\mathrm{HIF}-\alpha)$ were determined by western blotting. We found that knockout of EFNA3 notably decreased the protein expression of p-FAK and TNF- $\alpha$ compared to the control groups (Fig. 5A and B), while the protein expression levels of PI3K, GTPase, integrins, VEGF and HIF- $\alpha$ were significantly increased (Figs. 6-8). All these changes were beneficial to the progression of the tumor. On the contrary, overexpression of EFNA3 significantly upregulated the protein expression of p-FAK and TNF- $\alpha$ compared to the control groups (Fig. 5A and B), yet the protein levels of PI3K, GTPase, integrins, VEGF and HIF- $\alpha$ were notably reduced (Figs. 6-8). These data indicated that knockout and overexpression of EFNA3 improved and inhibited progression of MPNST cells, respectively. These data suggest that EFNA3 may function as a tumor suppressor in MPNST. 


\section{Discussion}

Ephrin ligands and their Eph receptors have been proven to play a crucial role in mediating a wide range of biological activities, such as angiogenesis, cell segregation, cell adhesion, shape and motility. As several of these processes are known to go awry during tumorigenesis and metastasis, Eph/ephrin signaling has been identified to play a role in many human cancers, such as lung, breast and prostate cancers, as well as melanoma and leukemia (9). Ephrin-A3 (EFNA3) is a GPI-anchored membrane protein and is widely expressed in human organisms, such as skeletal muscle, spleen, thymus, prostate, testis, and ovary (27). EFNA3 has been proven to play an important role in the guidance of various types of axons in the developing nervous system (28-30) and in the control of dendritic spine morphology (31). EFNA3 has also been proposed to be associated with some cancers. Iiizumi et al found that EFNA3 facilitated the growth of pancreatic cancer cells (32). Georgiou et al indicated that EFNA3 served as an angiogenesis-specific gene and its expression was upregulated in patients with breast cancer (33). Our previous study indicated that EFNA3 may play a part in the process of miR-210 promotion of growth and invasion of MPNST (1). Subsequently, the functions of EFNA3 in MPNST were investigated in this study.

In the present study, we investigated the function of EFNA3 by gain- and loss-of-function strategies. EFNA3-TALENs and Lv-EFNA3 were transfected into MPNST cells to knock out and overexpress EFNA3, respectively. The EFNA3 mRNA and protein levels were determined by RT-PCR and western blotting, and the results indicated that EFNA3 gene in ST88-14 and sNF96.2 cells were effectively knocked out by EFNA3-TALENs suggesting the promising application of TALENs in cancer associated gene function study.

We further investigated the performance of ST88-14 and sNF96.2 cells with EFNA3 present or knockout, on cell viability. The results suggested that knockout of EFNA3 significantly promoted the viability of MPNST cells even when miR-210 was pre-upregulated. Notably, our previous study showed that miR-210 promotes viability and proliferation of MPNST cells through negative regulation of EFNA3 (1). In the present study, although the MPNST cells were all transfected with pre-miR210, the viabilities still increased in ST88-14 and SNF96.2 cells when EFNA3 was further knocked out, implying that EFNA3 interfered in the viability of MPNST cells more directly than miR-210. Subsequently, the invasiveness of ST88-14 and sNF96.2 cells transfected with EFNA3-TALENs or pre-miR210 or both recombinant vectors were determined. Although the invasiveness of all transfected MPNST cell lines was increased, the enhancement degree was different in each. The enhancement degree of the invasiveness in the MPNST cells that were transfected with both EFNA3-TALENs and pre-miR-210 was greater than that of EFNA3-TALENs transfected cells but it was weaker than that of pre-miR-210 transfected cells. These data suggest that EFNA3 may not be the only target gene of miR-210. It was also consistent with the findings in our previous study, in which the ZNF462 gene was also indicated as a potential target of miR-210 (1). Besides, Fasanaro et al also identified some other targets of miR-210, including E2F3, MNT, APC, ACVR1B and CDK10, which were also demonstrated to be tumor suppressors (34).
Angiogenesis is always closely associated with tumor growth and metastasis. FAK signaling has been shown to promote angiogenesis in embryonic development as well as various physiological and disease processes in adult organism, including tumor angiogenesis (35). In addition, FAK signaling members and angiogenic factors have been implicated in tumorigenesis with regards to the Eph/ephrin axis. A study by Miao et al showed that EphA2 stimulation with ephrin-A1 leads to the recruitment of the protein tyrosine phosphatase SHP-2 to EphA2, followed by dephosphorylation of FAK and paxillin (36). Brantley et al validated the complementary expression of EphA2 in tumor blood vessel endothelium and ephrin-A1 in tumor cells as the first functional evidence of type-A Eph receptor regulation of pathogenic angiogenesis in tumors (37). Accordingly, the expression level of FAK signaling proteins and angiogenic factors were determined by western blotting to further clarify the potential molecular mechanisms underlying EFNA3-induced tumor angiogenesis in the present study. The results indicated that knockout of EFNA3 significantly decreased the expression of p-FAK. However, the expression of PI3K, GTPase and intergins were increased (Fig. 5). These suggested that FAK signaling was negatively regulated by phosphorylation of FAK. Generally, the FAK signaling is triggered by FAK phosphorylation that led to actin reorganization through downstream PI3K and GTPase activation (38). In addition, FAK activation has been linked to integrin clustering and is considered a critical step in the initiation of cell migration (37). This process seems to be positively regulated by FAK phosphorylation. However, phosphorylation of FAK at its tyrosine phosphoacceptor site Tyr-407 has been reported to negatively regulate kinase activity and cell migration (39). VEGF is a signal protein produced by cells that stimulate vasculogenesis and angiogenesis. It is part of the system that restores the oxygen supply to tissues when blood circulation is inadequate. When VEGF is overexpressed, it can contribute to cancer angiogenesis. HIF- $\alpha$ is the protein which plays an essential role in cellular and systemic responses to tumor mediated hypoxia. TNF- $\alpha$ is a member of a group of cytokines which is able to induce fever, apoptotic cell death, cachexia, inflammation and to inhibit tumorigenesis. VEGF, HIF- $\alpha$ and TNF- $\alpha$ are known as angiogenic factors which are closely related with tumorigenesis and angiogenesis. We also found that knockout of EFNA3 significantly inhibited the expression of TNF- $\alpha$ yet notably promoted the expression of VEGF and HIF- $\alpha$. These findings suggest that angiogenesis in MPNST cells was activated and may promote tumor metastasis when EFNA3 is knocked out. On the contrary, the expression of angiogenic factors was inversely different when EFNA3 was upregulated. Collectively, our results indicated that knockout of EFNA3 by TALENs may contribute to the development and progression of MPNST and this effect may be associated with increased viability and invasiveness, at least in part, via promoting angiogenesis. Moreover, overexpression of EFNA3 was able to inhibit the progression of MPNST.

In summary, we demonstrated that EFNA3 serves as a tumor suppressor in MPNST cells and it may play a critical role in the FAK signaling and VEGF-associated tumor angiogenesis pathway. These findings may not only facilitate better understanding of MPNST pathogenesis, but also suggest EFNA3 as a promising target for MPNST treatment. 


\section{References}

1. Wang Z, Yin B, Wang B, Ma Z, Liu W and Lv G: MicroRNA-210 promotes proliferation and invasion of peripheral nerve sheath tumor cells targeting EFNA3. Oncol Res 21: 145-154, 2014

2. Itani S, Kunisada T, Morimoto Y, Yoshida A, Sasaki T, Ito S, Ouchida M, Sugihara S, Shimizu K and Ozaki T: MicroRNA-21 correlates with tumorigenesis in malignant peripheral nerve sheath tumor (MPNST) via programmed cell death protein 4 (PDCD4). J Cancer Res Clin Oncol 138: 1501-1509, 2012.

3. Doorn PF, Molenaar WM, Buter J and Hoekstra HJ: Malignant peripheral nerve sheath tumors in patients with and without neurofibromatosis. Eur J Surg Oncol 21: 78-82, 1995.

4. Ingham S, Huson SM, Moran A, Wylie J, Leahy M and Evans DG: Malignant peripheral nerve sheath tumours in NF1: improved survival in women and in recent years. Eur J Cancer 47: 2723-2728, 2011.

5. Evans DG, Baser ME, McGaughran J, Sharif S, Howard E and Moran A: Malignant peripheral nerve sheath tumours in neurofibromatosis 1. J Med Genet 39: 311-314, 2002.

6. Héroult M, Schaffner F and Augustin HG: Eph receptor and ephrin ligand-mediated interactions during angiogenesis and tumor progression. Exp Cell Res 312: 642-650, 2006.

7. Kuijper S, Turner CJ and Adams RH: Regulation of angiogenesis by Eph-ephrin interactions. Trends Cardiovasc Med 17: 145-151, 2007.

8. Irie F, Okuno M, Matsumoto K, Pasquale EB and Yamaguchi Y: Heparan sulfate regulates ephrin-A3/EphA receptor signaling. Proc Natl Acad Sci USA 105: 12307-12312, 2008.

9. Surawska H, Ma PC and Salgia R: The role of ephrins and Eph receptors in cancer. Cytokine Growth Factor Rev 15: 419-433, 2004.

10. Böhme B, Holtrich U, Wolf G, Luzius H, Grzeschik KH, Strebhardt K and Rübsamen-Waigmann H: PCR mediated detection of a new human receptor-tyrosine-kinase, HEK 2. Oncogene 8 : $2857-2862,1993$

11. Fox BP and Kandpal RP: Invasiveness of breast carcinoma cells and transcript profile: Eph receptors and ephrin ligands as molecular markers of potential diagnostic and prognostic application. Biochem Biophys Res Commun 318: 882-892, 2004

12. Walker-Daniels J, Coffman K, Azimi M, Rhim JS, Bostwick DG Snyder P, Kerns BJ, Waters DJ and Kinch MS: Overexpression of the EphA2 tyrosine kinase in prostate cancer. Prostate 41: 275-280, 1999 .

13. Ambros V: The functions of animal microRNAs. Nature 431: 350-355, 2004

14. Qiu S, Lin S, Hu D, Feng Y, Tan Y and Peng Y: Interactions of miR-323/miR-326/miR-329 and miR-130a/miR-155/miR-210 as prognostic indicators for clinical outcome of glioblastoma patients. J Transl Med 11: 10, 2013.

15. Redova M, Poprach A, Besse A, Iliev R, Nekvindova J, Lakomy R, Radova L, Svoboda M, Dolezel J, Vyzula R, et al: MiR-210 expression in tumor tissue and in vitro effects of its silencing in renal cell carcinoma. Tumour Biol 34: 481-491, 2013.

16. Puisségur MP, Mazure NM, Bertero T, Pradelli L, Grosso S, Robbe-Sermesant K, Maurin T, Lebrigand K, Cardinaud B, Hofman V, et al: miR-210 is overexpressed in late stages of lung cancer and mediates mitochondrial alterations associated with modulation of HIF-1 activity. Cell Death Differ 18: 465-478, 2010.

17. Hong L, Yang J, Han Y, Lu Q, Cao J and Syed L: High expression of miR-210 predicts poor survival in patients with breast cancer: a meta-analysis. Gene 507: 135-138, 2012.

18. Rothé F, Ignatiadis M, Chaboteaux C, Haibe-Kains B, Kheddoumi N, Majjaj S, Badran B, Fayyad-Kazan H, Desmedt C, Harris AL, et al: Global microRNA expression profiling identifies miR-210 associated with tumor proliferation, invasion and poor clinical outcome in breast cancer. PLoS One 6: e20980, 2011

19. Chan SY and Loscalzo J: MicroRNA-210: a unique and pleiotropic hypoxamir. Cell Cycle 9: 1072-1083, 2010.

20. Cui H, Grosso S, Schelter F, Mari B and Krüger A: On the prometastatic stress response to cancer therapies: evidence for a positive co-operation between TIMP-1, HIF-1 $\alpha$, and miR-210. Front Pharmacol 3: 134, 2012.
21. Presneau N, Eskandarpour M, Shemais T, Henderson S, Halai D, Tirabosco R and Flanagan AM: MicroRNA profiling of peripheral nerve sheath tumours identifies miR-29c as a tumour suppressor gene involved in tumour progression. Br J Cancer 108: 964-972, 2013.

22. Kim YK, Wee G, Park J, Kim J, Baek D, Kim JS and Kim VN: TALEN-based knockout library for human microRNAs. Nat Struct Mol Biol 20: 1458-1464, 2013.

23. Boch J: TALEs of genome targeting. Nat Biotechnol 29: 135-136, 2011.

24. Arocho A, Chen B, Ladanyi M and Pan Q: Validation of the 2-DeltaDeltaCt calculation as an alternate method of data analysis for quantitative PCR of BCR-ABL P210 transcripts. Diagn Mol Pathol 15: 56-61, 2006.

25. Doyle EL, Booher NJ, Standage DS, Voytas DF, Brendel VP, Vandyk JK and Bogdanove AJ: TAL Effector-Nucleotide Targeter (TALE-NT) 2.0: tools for TAL effector design and target prediction. Nucleic Acids Res 40: W117-W122, 2012.

26. Sanjana NE, Cong L, Zhou Y, Cunniff MM, Feng G and Zhang F: A transcription activator-like effector toolbox for genome engineering. Nat Protoc 7: 171-192, 2012.

27. Pulkkinen K, Malm T, Turunen M, Koistinaho $J$ and Ylä-Herttuala S: Hypoxia induces microRNA miR-210 in vitro and in vivo ephrin-A3 and neuronal pentraxin 1 are potentially regulated by miR-210. FEBS Lett 582: 2397-2401, 2008.

28. Kullander K, Mather NK, Diella F, Dottori M, Boyd AW and Klein R: Kinase-dependent and kinase-independent functions of EphA4 receptors in major axon tract formation in vivo. Neuron 29: 73-84, 2001

29. Cutforth T, Moring L, Mendelsohn M, Nemes A, Shah NM, Kim MM, Frisén J and Axel R: Axonal ephrin-As and odorant receptors: coordinate determination of the olfactory sensory map. Cell 114: 311-322, 2003

30. Cang J, Kaneko M, Yamada J, Woods G, Stryker MP and Feldheim DA: Ephrin-as guide the formation of functional maps in the visual cortex. Neuron 48: 577-589, 2005

31. Murai KK, Nguyen LN, Irie F, Yamaguchi Y and Pasquale EB: Control of hippocampal dendritic spine morphology through ephrin-A3/EphA4 signaling. Nat Neurosci 6: 153-160, 2003.

32. Iiizumi M, Hosokawa M, Takehara A, Chung S, Nakamura $T$, Katagiri T, Eguchi H, Ohigashi H, Ishikawa O, Nakamura Y, et al: EphA4 receptor, overexpressed in pancreatic ductal adenocarcinoma, promotes cancer cell growth. Cancer Sci 97: 1211-1216, 2006.

33. Georgiou GK, Igglezou M, Sainis I, Vareli K, Batsis H, Briasoulis E and Fatouros M: Impact of breast cancer surgery on angiogenesis circulating biomarkers: a prospective longitudinal study. World J Surg Oncol 11: 213, 2013.

34. Fasanaro P, Greco S, Lorenzi M, Pescatori M, Brioschi M, Kulshreshtha R, Banfi C, Stubbs A, Calin GA, Ivan M, et al: An integrated approach for experimental target identification of hypoxia-induced miR-210. J Biol Chem 284: 35134-35143, 2009.

35. Zhao J and Guan JL: Signal transduction by focal adhesion kinase in cancer. Cancer Metastasis Rev 28: 35-49, 2009.

36. Miao H, Burnett E, Kinch M, Simon E and Wang B: Activation of EphA2 kinase suppresses integrin function and causes focaladhesion-kinase dephosphorylation. Nat Cell Biol 2: 62-69, 2000.

37. Brantley DM, Cheng N, Thompson EJ, Lin Q, Brekken RA, Thorpe PE, Muraoka RS, Cerretti DP, Pozzi A, Jackson D, et al: Soluble Eph A receptors inhibit tumor angiogenesis and progression in vivo. Oncogene 21: 7011-7026, 2002.

38. Kallergi G, Agelaki S, Markomanolaki H, Georgoulias V and Stournaras C: Activation of FAK/PI3K/Racl signaling controls actin reorganization and inhibits cell motility in human cancer cells. Cell Physiol Biochem 20: 977-986, 2007.

39. Lim Y, Park H, Jeon J, Han I, Kim J, Jho EH and Oh ES: Focal adhesion kinase is negatively regulated by phosphorylation at tyrosine 407. J Biol Chem 282: 10398-10404, 2007. 